\title{
REALIDADE VIRTUAL: PERCEPÇÕES DOS STAKEHOLDERS
}

Clara Silva - clarabeatrizfidelis623@gmail.com

Instituto Federal de Educação, Ciência e Tecnologia do Rio Grande do Norte

Natal - RN

Josyanne Giesta - josyanne.giesta@ifrn.edu.br

Instituto Federal de Educação, Ciência e Tecnologia do Rio Grande do Norte

Natal - RN

Anny Costa - annycarolinesc@outlook.com

Instituto Federal de Educação, Ciência e Tecnologia do Rio Grande do Norte

Natal - RN

Karine Morais - karinemorais2002@gmail.com

Instituto Federal de Educação, Ciência e Tecnologia do Rio Grande do Norte

Natal - RN

Thalita Costa - thalitagiesta@yahoo.com.br

Universidade Federal do Rio Grande do Norte

Natal - RN

\section{Resumo}

Realidade Virtual (RV) é uma interface que permite a criação de um ambiente por meio de softwares que proporcionam a comunicação interativa entre o usuário e seu computador, englobando programas de modelação e representação tridimensional. Além disso, essa tecnologia vem apresentando uma acelerada utilização em diferentes áreas, sobretudo para simulação de espaços projetados. A tecnologia RV tem como finalidade viabilizar a sensação máxima de realidade de forma que seja possível visualizar, interagir e manipular seus objetos em tempo real. A partir dos inúmeros avanços tecnológicos voltados ao setor da Arquitetura, Engenharia e Construção (AEC), a RV poderá atuar como ferramenta facilitadora da relação entre utilizador e máquina, por possibilitar a inserção e interação virtual do indivíduo no ambiente a ser construído. Nesse aspecto, o presente artigo objetiva conhecer as percepções dos stakeholders com relação a utilização da RV na área de AEC. Os métodos adotados foram o estudo de caso e o comparativo entre estudos de caso. Os resultados indicam que projetistas de instalações, alunos de Edificações e proprietários identificam vantagens na visualização de modelo 3D em óculos estereoscópicos. Nesse contexto, pode-se concluir que a utilização da RV facilita a compreensão dos projetos.

Palavras-chave: Building Information Modeling. RV. Óculos estereoscópicos. 


\section{VIRTUAL REALITY: STAKEHOLDERS PERCEPTIONS}

\section{Abstract:}

Virtual Reality (VR) is an interface that allows the creation of an environment through softwares that provide interactive communication between the user and his computer, including modeling and three-dimensional representation programs. In addition, this technology has been showing accelerated use in different areas, especially for simulating projected spaces. The purpose of VR technology is to enable the maximum sensation of reality so that it is possible to visualize, interact and manipulate the objects in real time. Based on the countless technological advances aimed at the sector of Architecture, Engineering and Construction (AEC), VR can act as a facilitator of the relationship between user and machine, as it allows the virtual insertion and interaction of the individual in the environment to be built. In this regard, this article aims to understand the perceptions of stakeholders regarding the use of VR in the area of AEC. The methods adopted were the case study and the comparison between case studies. The results indicate that installation designers, Buildings students, and owners identify advantages in viewing the 3D model in stereoscopic glasses. In this context, it can be concluded that the use of VR facilitates the understanding of projects.

Keywords: Building Information Modeling. VR. Stereoscopic glasses.

\section{INTRODUÇÃO}

O Building Information Modeling (BIM), vem desde 2017 sendo oficialmente estimulado pelo governo federal brasileiro, através de decretos federais. Recentemente o decreto $\mathrm{N}^{\circ}$ 10.306, estabeleceu a utilização do BIM na execução direta e indireta de obras e serviços de engenharia realizada pelos órgãos e pelas entidades da administração pública federal (BRASIL, 2020).

Estudos apontam múltiplas vantagens da implementação do BIM, no entanto, é relevante considerar que é necessário definir o objetivo para então poder selecionar o uso do modelo (MU) que será aplicado. Nesse aspecto, Bime Initiative Research for Innovation apresenta 73 domínios MUs, que correspondem aos usos possíveis para cada modelo. O uso Simulação da Realidade Virtual (4240) significa o uso do modelo em um ambiente imersivo no qual os usuários experimentam locais, objetos e processos simulados. A Realidade Virtual (RV) pode exigir "imersão" total em salas de projeção múltipla (CAVE) e/ou através de óculos estereoscópicos e outros equipamentos especializados.

A RV teve o seu início na indústria de simulação, com os simuladores de voo que a Força Aérea dos Estados Unidos passou a construir depois da Segunda Guerra Mundial (JACOBSON, 1994). No entanto, a expressão "Realidade Virtual" foi elaborada no final da década de 1980 por Jaron Lanier, cientista da computação e artista, que possibilitou afluir dois conceitos antagônicos em um novo conceito, diferindo as simulações tradicionais feitas por computador das simulações envolvendo múltiplos usuários em um ambiente compartilhado (ARAÚJO, 1996). Freitas (2008) acrescenta que a RV pode ser descrita como um conjunto de tecnologias que permite criar ambientes gráficos que simulam a realidade ou a realidade projetada.

Com a RV o indivíduo é capaz de vivenciar mundos ainda inexistentes fisicamente, por meio de equipamentos que o faz sentir a nítida impressão de estar no ambiente gerado em computador, proporcionando uma "interação natural" com os ambientes sintéticos por ele 
gerado. Dessa forma, o poder de imersão oferecido é um fator importante, porque torna possível vivenciar o espaço concebido, ao invés de apenas visualizá-lo através da tela de um computador.

O BIM e seus modelos de uso (MUs) envolvem muitas pessoas, as quais são conhecidas como stakeholders. A identificação desses é importante, pois se configura como o primeiro passo do planejamento estratégico de implantação do BIM na cadeia produtiva da construção civil (BÖES; LIMA; BARROS NETO, 2018). Nesse mesmo sentido, para a utilização da RV é importante identificar e compreender os stakeholders. Suzuki e Santos (2015), apresentam 5 stakeholders: proprietário, incorporador, construtor, projetista e outros.

Diante desse cenário, o presente artigo tem como objetivo conhecer as percepções dos stakeholders quanto a aplicação da Realidade Virtual no mercado da área de Arquitetura, Engenharia e Construção (AEC).

\section{MÉTODO}

Objetivando apresentar as percepções dos stakeholders com relação a aplicação da Realidade Virtual, realizou-se a presente pesquisa, que teve a utilização de dois métodos de pesquisa: estudo de caso e estudo comparativo.

O estudo de caso, que segundo Dresch; Lacerda e Antunes Júnior (2015) se configura como uma pesquisa empírica que busca melhor compreender um fenômeno contemporâneo, normalmente complexo, no seu contexto real, foi estruturado em 6 etapas (Figura 1): a) definição da estrutura conceitual; b) planejamento do caso; c) condução do teste piloto; d) coleta dos dados; e) análise dos dados; f) geração do relatório.

Figura 1 - Etapas de um estudo de caso

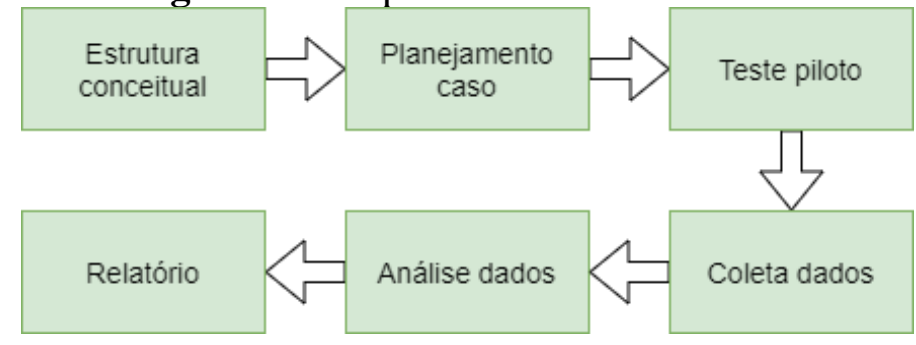

Fonte - Os Autores

$\mathrm{Na}$ etapa de definição da estrutura conceitual, foi realizada uma revisão da literatura, e para tanto, a primeira tarefa foi identificar as palavras-chave (ECHER, 2001) e depois definir as bases de dados. O planejamento do caso consistiu em selecionar a unidade de análise e elaborar o protocolo para a coleta de dados. O protocolo tem como objetivo, segundo Yin (2009) orientar o pesquisador nas atividades de coleta de dados, definindo as regras a serem seguidas. Deve constar dos instrumentos de pesquisa e os procedimentos para coleta dos dados.

A etapa do teste piloto foi realizada objetivando testar o protocolo de pesquisa, de modo a fazer os ajustes necessários, tanto com relação ao tempo necessário para a aplicação, como as dificuldades de interpretação das perguntas.

Para a etapa de coleta de dados foi elaborado um questionário com perguntas fechadas, que restringem as respostas, facilitando a análise dos dados em razão da objetividade (DRESCH; LACERDA; ANTUNES JÚNIOR, 2015).

$\mathrm{Na}$ etapa de análise dos dados, os resultados obtidos com os questionários foram analisados de modo a serem formuladas as conclusões para o relatório final. 
O estudo comparativo foi realizado entre o estudo de caso realizado e outros dois estudos de caso já publicados (Quadro 1), onde cada um destes focou em stakeholders diferentes:

Quadro 1 - Trabalhos selecionados para o estudo comparativo

\begin{tabular}{|l|l|l|}
\hline Estudo de caso & Estudo 01 & Estudo 02 \\
\hline Autores & $\begin{array}{l}\text { Giesta, J. P.; Lira, E. C. F. da S.; } \\
\text { Costa, T. G.; Silva, J. A. A. da e } \\
\text { Costa Neto, A. }\end{array}$ & $\begin{array}{l}\text { Giesta, J. P.; Costa, T. G.; Silva, J. } \\
\text { A. A. da; Lira, E. C. F. da S. }\end{array}$ \\
\hline Título & $\begin{array}{l}\text { Evolução da tecnologia de Realidade } \\
\text { Virtual: estudo de caso no ensino de } \\
\text { Edificações no IFRN CNAT }\end{array}$ & $\begin{array}{l}\text { Implementação da Realidade } \\
\text { Virtual no mercado de trabalho da } \\
\text { área AEC - Estudo de caso }\end{array}$ \\
\hline Evento & $\begin{array}{l}\text { XIV CONGIC - Congresso de } \\
\text { Iniciação Científica do IFRN }\end{array}$ & $\begin{array}{l}\text { III CONACED - Congresso } \\
\text { Nacional de Construção de } \\
\text { Edifícios }\end{array}$ \\
\hline Ano & 2018 & 2018 \\
\hline Stakeholders & Alunos do curso de Edificações & Projetistas de instalações \\
\hline
\end{tabular}

Fonte - Os Autores

\section{ESTUDO DE CASO}

Para o desenvolvimento do estudo de caso, foi preciso definir o público alvo, que deveria ser composto por cinco pessoas leigas, que não tivessem nenhum conhecimento acerca da área de AEC, ou seja, o público usuário final das edificações (proprietários). Para analisar e interpretar os resultados obtidos com a aplicação dos questionários foram adotados as seguintes etapas (Figura 2): 1) Renderização dos ambientes presentes no projeto arquitetônico; 2) Plotagem da planta baixa do projeto; 3 ) Inserção dos ambientes em óculos de Realidade Virtual; 4) Anotação das respostas dos questionários aplicados.

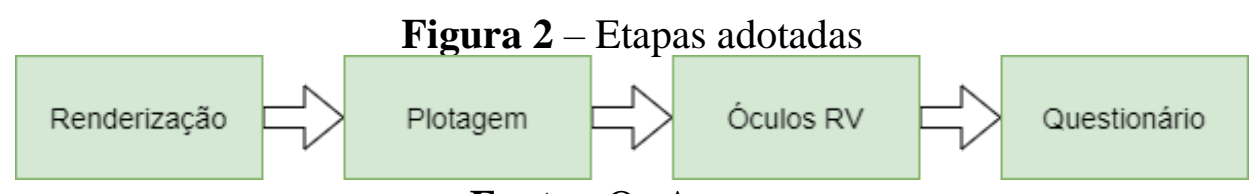

Fonte - Os Autores

Como primeira etapa, após a seleção dos entrevistados, foi necessário o desenvolvimento de um projeto arquitetônico para a plotagem em $2 \mathrm{D}$ e para a renderização em 3D para aplicação nos óculos estereoscópicos. Para a modelagem foi utilizado o software Autodesk Revit (2019), por ser do conhecimento das pesquisadoras e estar disponível no laboratório do Grupo de Estudos e Pesquisa em Integração de Projetos (GIP).

Para a renderização, foi utilizado o Revit Cloud, que tem como benefícios o armazenamento em nuvem que se apossa da memória disponibilizada pela Autodesk e a renderização ocorrer em segundo plano permitindo que o usuário use sua máquina enquanto ela ocorre. O Revit Cloud disponibiliza links e códigos que podem ser compartilhados e que levam os ambientes virtuais para os smartphones podendo assim serem exibidos através dos óculos de Realidade Virtual. Os três óculos RV utilizados na pesquisa foram adquiridos pelos pesquisadores do GIP, sendo óculos estereoscópicos VX Case, que funcionam em conjunto com smartphone (Figura 3). 


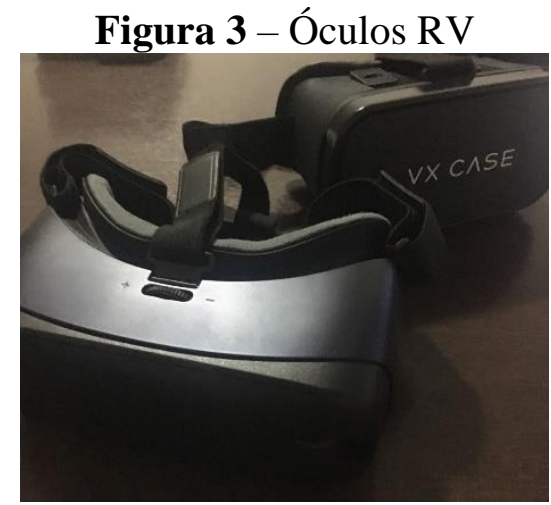

Fonte - Os Autores

O projeto elaborado (Figura 4), corresponde a uma residência unifamiliar, com oito ambientes: 1) Cozinha; 2) Lavanderia; 3) Quarto de casal; 4) Banheiro social; 5) Sala de jantar; 6) Sala de estar; 7) Área de lazer; 8) Hall. Os ambientes foram disponibilizados em duas versões para os entrevistados, isto é, plotado e renderizado, a fim de escolherem a versão com a qual possuem mais afinidade e entendimento.

Figura 4 - Planta baixa 2D e Renderização 3D nos óculos RV
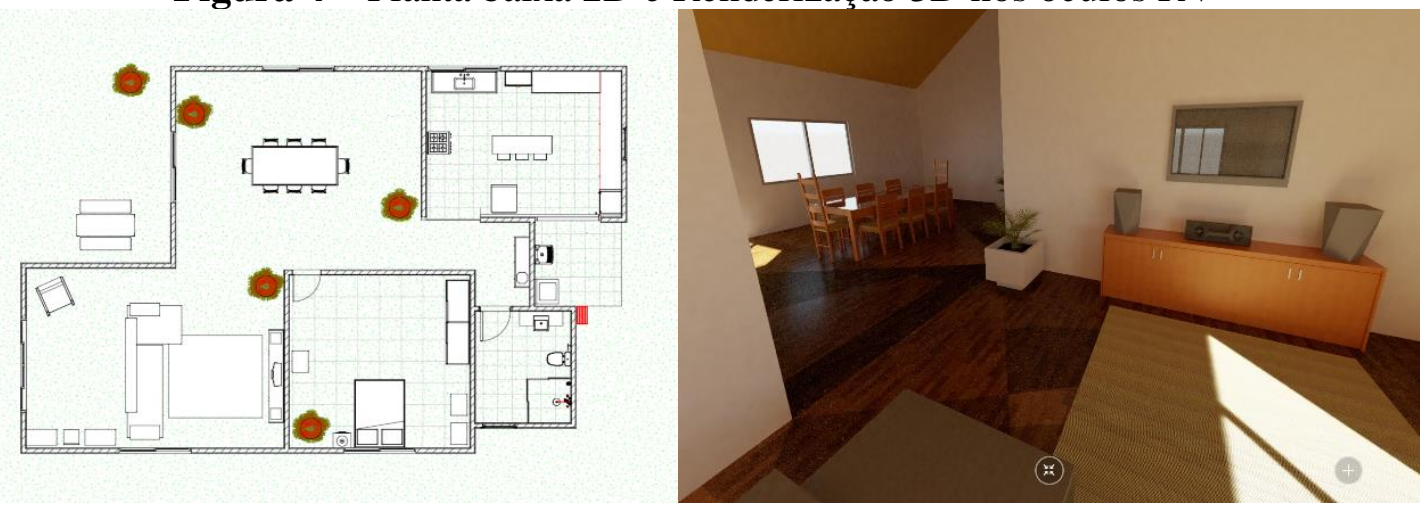

Fonte - Os Autores

A planta 2D plotada não proporciona a visualização dos materiais, quase nenhum detalhamento e alguns objetos não conseguem aparecer. Diferentemente, do projeto renderizado nos óculos estereoscópicos, o qual unifica todos esses elementos com o objetivo de melhorar a experiência do usuário. Com a RV, é possível aplicar recursos como texturas, iluminação na superfície ou modificar a aparência do objeto. A maior vantagem de aplicar a tecnologia de RV é o fato de poder escolher uma cor para esses parâmetros ou uma textura que deve ser importada e armazenada antes do processo de criação de materiais.

\section{ANÁLISE DOS DADOS DO ESTUDO DE CASO}

A parte inicial da aplicação dos questionários consistiu em uma breve caracterização do entrevistado, a qual resultou em que $20 \%$ correspondem ao gênero feminino, possuem entre 41 e 50 anos e têm graduação; os $80 \%$ restantes pertencem ao gênero masculino, possuem idade máxima de 20 anos e estão cursando o ensino médio.

Quando questionados a respeito da qualidade de visualização das cores, materiais e texturas do projeto apresentado em RV, 60\% dos participantes considerou excelente, $40 \%$ considerou boa e nenhum achou ruim. Tal resultado indica que o uso da RV na arquitetura é viável, como mecanismo para facilitar a compreensão dos ambientes antes da construção e, 
consequentemente, ampliar as possibilidades de visualização do cliente, oferecendo maneiras inovadoras de experimentar e entender o projeto muito antes da execução do mesmo.

Com relação ao questionamento sobre a segurança na compra do imóvel, todos os entrevistados disseram se sentir mais seguros para realizar uma compra visualizando o projeto renderizado e inserido nos óculos de RV. Com o projeto 3D inserido nos óculos RV, o cliente final pode falar para o profissional o que prefere mudar, evitando futuras frustrações ou erros. Os óculos RV utilizados são responsáveis por introduzir as pessoas no ambiente virtual, eles conseguem transmitir a sensação de estar fisicamente dentro do ambiente. Esse é o principal motivo pelo qual é oportuno utilizar a RV na apresentação de projetos de arquitetura. Nesse sentido, Heidrich e Pereira (2004) apontam que o uso da visualização de modelos tridimensionais digitais, em Realidade Virtual, pode substituir o conhecimento da linguagem técnica tradicional de plantas e fachadas de forma satisfatória (SOPEÑA et al., 2015).

Por fim, é importante salientar que os resultados apontaram que $40 \%$ dos participantes afirmaram não ter conhecimento prévio sobre Realidade Virtual. Aliando essa informação a anterior, a qual mostra que todos os entrevistados sentiram maior segurança através do projeto renderizado, é possível perceber que pessoas com baixo nível de entendimento na área de AEC, reconhecem os benefícios de visualizar um projeto com aplicação da RV. Tais resultados corroboram com o empoderamento vivenciado pelos moradores do bairro Frei Damião, na cidade de Pau dos Ferros (RN) durante a apresentação da proposta de Intervenção Urbanística para o bairro através de óculos de Realidade Virtual (GIESTA et al., 2019).

\section{COMPARATIVO DOS CASOS}

Uma análise comparativa entre as percepções dos stakeholders sobre a utilização de Realidade Virtual pode ser visualizada no Quadro 2:

Quadro 2 - Comparativo dos casos

(continua)

\begin{tabular}{|c|c|c|c|}
\hline Área & Mercado de trabalho & Ensino & Leigos \\
\hline Stakeholders & $\begin{array}{ll}\text { Projetistas } & \text { de } \\
\text { instalações } & \\
\text { hidrossanitárias } & \\
\end{array}$ & $\begin{array}{l}\text { Alunos do curso de } \\
\text { Edificações }\end{array}$ & $\begin{array}{l}\text { Usuário } \\
\text { (proprietário) }\end{array}$ \\
\hline Projeto & $\begin{array}{l}\text { Banheiros fem. e mas. } \\
\text { de Instituição de Ensino } \\
\text { com } 47,10 \mathrm{~m}^{2}\end{array}$ & $\begin{array}{l}\text { Residência duplex com } \\
283,65 \mathrm{~m}^{2}\end{array}$ & $\begin{array}{l}\text { Residência térrea com } 8 \\
\text { ambientes }\end{array}$ \\
\hline Proposta & $\begin{array}{l}\text { a) Projeto plotado } \\
\text { em } 2 \mathrm{D} \mathrm{CAD} \\
\text { b) } 3 \mathrm{D} \text { em RV }\end{array}$ & $\begin{array}{l}\text { a) Projeto plotado } \\
\text { em } 2 \mathrm{D} \mathrm{CAD} \\
\text { b) } 3 \mathrm{D} \mathrm{em} \mathrm{RV} \\
\end{array}$ & $\begin{array}{l}\text { a) Projeto plotado } \\
\text { em } 2 \mathrm{D} \mathrm{CAD} \\
\text { b) } 3 \mathrm{D} \text { em } \mathrm{RV} \\
\end{array}$ \\
\hline Resu & $\begin{array}{l}\text { Os profissionais da área } \\
\text { apontaram que a } \\
\text { utilização da RV facilita } \\
\text { a compreensão do } \\
\text { projeto, diminuindo } \\
\text { erros. Indicaram } \\
\text { inclusive que seria } \\
\text { recomendado a inserção } \\
\text { da RV em aulas de } \\
\text { instalações. }\end{array}$ & $\begin{array}{l}\text { A maioria dos alunos } \\
\text { das } 3 \text { turmas } \\
\text { participantes } \\
\text { pesquisa considerou } \\
\text { que com relação a } \\
\text { visão/entendimento do } \\
\text { projeto a experiência } \\
\text { com RV apresenta } \\
\text { resultados melhores que } \\
\text { o projeto 2D plotado. }\end{array}$ & $\begin{array}{l}\text { A maioria dos } \\
\text { entrevistados classificou } \\
\text { como excelente a } \\
\text { visualização das texturas } \\
\text { e cores com a RV, bem } \\
\text { como afirmou que } \\
\text { sentem maior segurança } \\
\text { na compra de um imóvel } \\
\text { quando visualizam nos } \\
\text { óculos RV do que em } \\
\text { projeto 2D plotado. }\end{array}$ \\
\hline
\end{tabular}


Quadro 2 - Comparativo dos casos

\begin{tabular}{|l|l|l|lr|}
\hline Área & Mercado de trabalho & Ensino & Leigos \\
\hline Conclusões & A utilização do método & A introdução da & A aplicação da RV para \\
& imersivo no processo de & tecnologia RV como & o usuário final lhe \\
& elaboração dos projetos & uma ferramenta didática & proporciona & um \\
& de instalações & no processo de ensino- & empoderamento na \\
& hidrossanitárias & aprendizagem permite & tomada de decisões \\
& apresenta vantagens. & melhor compreensão e & quanto ao produto que \\
& & maior memória visual & está adquirindo. \\
& & & \\
& &
\end{tabular}

Fonte - Os Autores

\section{CONCLUSÃO}

O presente artigo buscou através de um estudo de caso conhecer as percepções de proprietários, pessoas leigas em termos de Arquitetura, Engenharia e Construção, com relação a utilização da Realidade Virtual para a apresentação de projetos, bem como comparar com as percepções obtidas em outros dois estudos de caso realizados, um com projetistas de instalações e outro com alunos de curso da área de AEC.

A análise dos dados coletados permitiu compreender que os 3 grupos de stakeholders pesquisados tem percepções semelhantes com relação à $R V$, todos identificam maior facilidade na interpretação do projeto quando inserido nos óculos estereoscópicos. Tais resultados vem confirmar a importância da utilização da tecnologia, como forma de evitar erros de interpretação no caso dos projetistas, facilitar a compreensão de conteúdos no caso dos alunos e aumentar o empoderamento na tomada de decisões com relação aos usuários finais da edificação.

Como limitações pode-se apontar o estudo de caso e o comparativo terem abordado apenas 3 stakeholders, a saber: projetistas de instalações, proprietários e alunos. Nesse contexto, tem-se como proposta para pesquisas futuras a realização de estudos de caso em canteiros de obras, de modo a conhecer a percepção dos operários com relação a utilização da Realidade Virtual dentro da obra; nas instituições de ensino, para ouvir os docentes e em escritórios para entrevistar projetistas das disciplinas de arquitetura e de estrutura.

\section{AGRADECIMENTOS}

Os autores agradecem ao Conselho Nacional de Desenvolvimento Científico e Tecnológico (CNPq) e a Pró-Reitoria de Pesquisa e Inovação (PROPI) do Instituto Federal de Educação, Ciência e Tecnologia do Rio Grande do Norte (IFRN), pelo apoio recebido no desenvolvimento da pesquisa.

\section{REFERÊNCIAS}

ARAÚJO, R. B. Especificação e análise de um sistema distribuído de realidade virtual. 1996. Tese de doutorado - Departamento de Engenharia de Computação e Sistemas Digitais, Escola Politécnica da Universidade de São Paulo, São Paulo, 1996. 
Bime Initiative Research for Innovation. Disponível em: <https://bimexcellence.org/files/211in-Model-Uses-Table.pdf>. Acesso em 11 ago. 2020.

BÖES, J. S., LIMA, M. M. X., BARROS NETO, J. P. Identificação dos stakeholders envolvidos no processo de desenvolvimento de projetos na cadeia da construção civil do estado do Ceará: o primeiro passo para adoção do BIM. In: ENCONTRO NACIONAL DE TECNOLOGIA DO AMBIENTE CONSTRUÍDO, 18., 2018, Foz do Iguaçu. Anais... Porto Alegre: ANTAC, 2018.

BRASIL. Decreto $n^{\circ} 10.306$, de 02 de abril de 2020. Dispõe sobre a utilização do Building Information Modelling - BIM ou Modelagem da Informação da Construção na execução direta ou indireta de obras e serviços de engenharia, realizada pelos órgãos e pelas entidades da administração pública federal, no âmbito da Estratégia Nacional de Disseminação do Building Information Modelling - Estratégia BIM BR. Diário Oficial da República Federativa do Brasil, Brasília, n. 65, Seção 1, p. 5-7, abr. 2020.

DRESCH, A.; LACERDA, D. P.; ANTUNES Jr., J. A. V. Design Science Research: Método de Pesquisa para Avanço da Ciência e Tecnologia. Porto Alegre: Bookman, 2015. 204 p.

ECHER, I. C. A revisão de literatura na construção do trabalho científico. Revista Gaúcha de Enfermagem, 22, 5-20. 2001.

FREITAS, M. R. LudosTop: Estratégia de jogos e Realidade Virtual com vistas ao desenvolvimento do pensamento lógico-matemático. 2008. Dissertação (Mestrado) Mestrado em Engenharia Elétrica, Universidade Federal de Uberlândia, Uberlândia, 2008.

GIESTA, J.P.; CAMPOS, T. M. da C. M.; MORAIS, K. da S.; COSTA, T. G. Relatos da disseminação do BIM por meio de uma experiência didática de extensão. In: ENCONTRO NACIONAL SOBRE O ENSINO DE BIM, 2019, Fortaleza. ENEBIM, 2019.

JACOBSON, L. Realidade virtual em casa. Rio de Janeiro: Berkeley, 1994.

SOPEÑA, S. M.; SILVA, A. B. A.; MEDVEDOVISKI, N. S.; KERKHOFF, H.V. O uso de tecnologias de Realidade Aumentada como estratégia de empoderamento In: ENCONTRO BRASILEIRO DE TECNOLOGIA DE INFORMAÇÃO E COMUNICAÇÃO NA CONSTRUÇÃO, 7., 2015, Recife. Anais... Porto Alegre: ANTAC, 2015.

SUZUKI, R. T.; SANTOS, E. T. Planejamento 4D no brasil: levantamento orientado à percepção de resultados pelos diversos "stakeholders" da construção. In: ENCONTRO BRASILEIRO DE TECNOLOGIA DE INFORMAÇÃO E COMUNICAÇÃO NA CONSTRUÇÃO, 7., 2015, Recife. Anais... Porto Alegre: ANTAC, 2015.

YIN, R. K. Case study research, design and methods (applied social research methods). Thousand Oaks. California: Sage Publications. 2009. 